\title{
A MONTE CARLO PROGRAM FOR GENERATING HADRONIC FINAL STATES IN ELECTRON-POSITRON ANNIHILATIONS
}

\author{
L. ANGELINI, L. NITTI, M. PELliCORO, G. PREPARATA \\ Dipartimento di Fisica, Universita di Bari, Italy \\ Istituto Nazionale di Fisica Nucleare, Sezione di Bari, Italy \\ and \\ G. VALENTI * \\ Department of Physics, University of Michigan, Ann Arbor, MI 48109, USA
}

Received 15 May 1984

\section{PROGRAM SUMMARY}

Title of program: EPOS

Catalogue number: ACDM

Program obtainable from: CPC Program Library, Queen's University of Belfast, $\mathbf{N}$. Ireland (see application form in this issue)

Computers on which the program is operable: CDC $7600 /$ CYBER $875 /$ VAX 780

Operating system: 7000 SCOPE 2.1.4 (CDC)/NOS-BE Level 587/VAX-VMS

\section{Programming language: FORTRAN}

High speed storage required: $50 \mathrm{Kwords}$ on CDC's and a peak value of $240 \mathrm{Kwords}$ on the VAX

No. of bits in a word: 60 (CDC), 32 (VAX)

No. of lines in combined program and test deck: 3938

Keywords: Monte Carlo, QGD, $\mathrm{e}^{+} \mathrm{e}^{-}$annihilation, hadron production

Nature of the physical problem

High energy electron-positron annihilations, at present investigated at PETRA and PEP and in the near future at SLC and LEP, have shown the production of a large number of

* On leave from Istituto Nazionale di Fisica Nucleare Sezione di Bologna, Italy. particles in the final state ( 30 or $\mathbf{4 0}$ particles are not uncommon at $W=35 \mathrm{GeV}$ ) [1]. The complexity of this phenomenon needs a theoretical framework for describing and understanding the basic physical mechanism involved.

The present program is an event generator of hadronic final states based on the Fire-String theory and has been shown to reproduce very well all the features experimentally observed [2].

Method of solution

The program is based on the Monte Carlo method and its generates events with weight $=1$ using the Fire-String theory [2]. The notion of Fire-String comes from the description of hadronic matter in terms of bound states (bags) of quarks and antiquarks in the theoretical framework named Quark-Geometro-Dynamics [3]. In this approach to the dynamics of high energy physics one can define a highly excited quark-antiquark bound state with a cylindrical structure, called Fire-string (FS). The evolution of the FS and its decay modes are responsible for the deposition of hadrons in the final states.

All these physical ideas have been implemented in the present program.

Restrictions on the complexity of the problem

The program describes quite well all the features of hadronic final states for energy ranging from 5 to $36 \mathrm{GeV}$, furthermore its yield can be extrapolated to sLC and LEP energies. A maximum production of $\mathbf{4 0 0}$ particles in the final state is allowed. It can, however, be easily modified to allow any number of particles.

Typical running time

The typical running time for a $W=30 \mathrm{GeV}$ event is: $19 \mathrm{~ms}$ on

0010-4655/85/\$03.30 (C) Elsevier Science Publishers B.V. (North-Holland Physics Publishing Division) 
the CYBER $875,21 \mathrm{~ms}$ on the CDC 7600 and about $200 \mathrm{~ms}$ on the VAX.

References

[1] For a recent review article on $\mathrm{e}^{+} \mathrm{e}^{-}$annihilations, see for instance: G. Wolf, DESY Report 82-077 (1982) and references therein.

\section{LONG WRITE-UP}

\section{Theoretical framework}

EPOS is a Monte Carlo program for the generation of hadronic final states in electron-positron annihilation at high energy $(W>5 \mathrm{GeV})$. The theoretical scheme is based on Quark-GeometroDynamics (QGD), where one can define highly excited quark-antiquark bound states with a cylindrical structure, called fire-Strings (FS) $[1,4]$. As we shall see, the notion of FS plays a central role in our model.

The basic idea of our approach is that the annihilation of an electron-positron pair will produce hadrons in the final state through a two stage process described schematically by

$\mathrm{e}^{+} \mathrm{e}^{-} \rightarrow \mathrm{FS} \rightarrow$ hadrons.

The two stages occur as follows:

1st stage: the electron-positron pair annihilates into a virtual $\gamma / Z^{0}$ which goes into a quark-antiquark pair: such a pair constitutes the initial FS;

2nd stage: the FS deposits hadrons in the final state through a multistep process arising from an iterative decay scheme.

Here we sketch briefly the main feature of the FS decay process. At each step the FS can choose three different decay modes, with assigned probabilities computed in the QGD framework. These probabilities depend on the FS mass (M) and flavours $[2,3]$. The decay modes are:

1) chain mode: FS decays into a FS and a vector meson;

2) tree mode: FS decays into two FS's;

3) baryon mode: FS decays into a baryon-antibaryon pair.

The chain mode is a peculiar high energy decay characterized by a strong transverse momentum cut-off. The tree mode occurs mainly when the FS
[2] L. Angelini, L. Nitti, M. Pellicoro, G. Preparata and G. Valenti, Riv. Nuovo Cimento 6 (1983) 1.

[3] G. Preparata, in: lepton Hadron Structure, ed. A. Zichichi (Academic Press, New York, London, 1975) p. 54.

mass is high ( $M>15 \mathrm{GeV})$, whereas the baryon mode is effective only at small values of $M$ (from the threshold to about $5 \mathrm{GeV}$ ). As the FS mass decreases due to the sequential decay process and goes below some lower bounds, a final step, we shall call "ENDFS", dumps all the mass into mesons. When this step is not possible because the FS mass is below the smallest two body threshold, the sequence is rejected and the program restarts the decay process of the same FS.

Finally, we remark that our model contains one unknown parameter $\beta$. This parameter normalizes the baryon mode with respect to all hadronic channels and it has been determined by a systematic comparison between the predictions of our model and the available experimental information [4].

The best value has been found to be $\beta=0.14$.

\section{Program description}

\subsection{Subroutine EPOS}

The subroutine EPOS controls the generation of a multiparticle event of weight $=1$ using the Monte Carlo technique, as schematically shown in fig. 1.

In what follows we give a brief description of the subroutines implemented.

\subsubsection{Subroutine FSGEN}

This routine generates the initial FS, using the inputs specified in

COMMON/EPIN/ECM,ICORAD,IFLAV,
IFQQB,QCT,QPHI




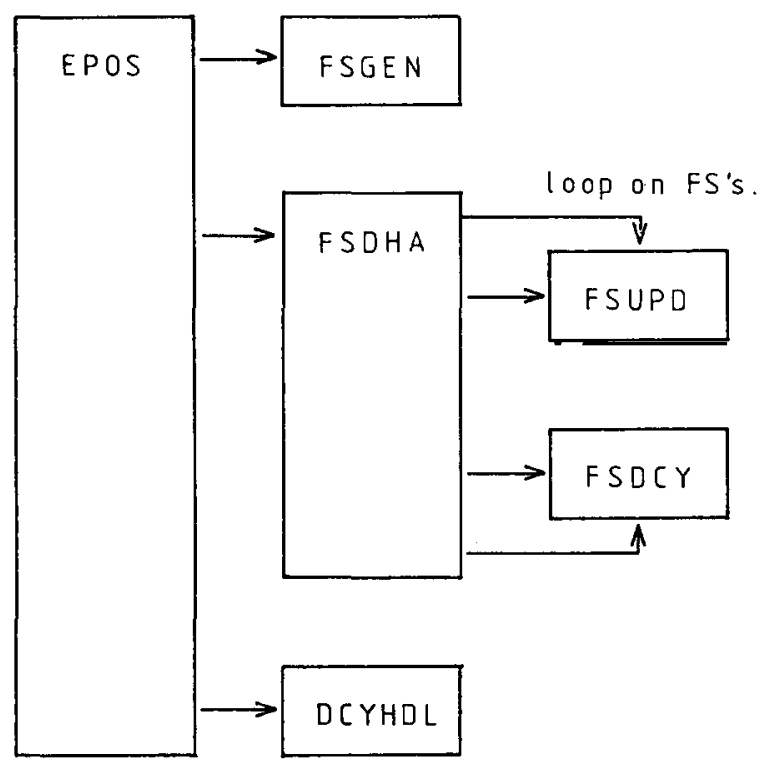

Fig. 1. Flow-chart of subroutine EPOS.

ECM is the total centre-of-mass energy of the initial state.

ICORAD is an option to include radiative corrections in the initial state (default is 0 );

$=0$ no,

$=1$ yes.

IFLAV option for flavour assignment to the initial quark-antiquark pair (default is $0)$;

$=0$ according to the square of the quark charge,

$=1,2,3,4,5$ for fixed flavour generation set by the user (see table 1).

IFQQB option to define the direction of the initial quark-antiquark pair (default is 0 );

$=0$ direction set by the user,

$=1$ according to QED at the first order,

$=2$ according to qED at the first order plus $\gamma / Z^{0}$ interference.

$\mathrm{QCT}=\cos (\theta), \mathrm{QPHI}=\phi$

where $\theta$ and $\phi$ are the polar and the azimuthal angles of the initial quark-antiquark pair with respect to the electron-positron line of flight in
Table 1

Quark parameters

\begin{tabular}{lllr}
\hline Flavour & Identifier & Mass $^{\text {a) }}$ & Charge \\
\hline u & 1 & 0.1 & $2 / 3$ \\
d & 2 & 0.1 & $-1 / 3$ \\
s & 3 & 0.469 & $-1 / 3$ \\
c & 4 & 1.86 & $2 / 3$ \\
b & 5 & 4.5 & $-1 / 3$ \\
ubar & 6 & 0.1 & $-2 / 3$ \\
dbar & 7 & 0.1 & $1 / 3$ \\
sbar & 8 & 0.469 & $1 / 3$ \\
cbar & 9 & 1.86 & $-2 / 3$ \\
bbar & 10 & 4.5 & $1 / 3$ \\
\hline
\end{tabular}

a) masses are given in $\mathrm{GeV}$.

the hadronic centre-of-mass (default values are: $\mathrm{QCT}=1, \mathrm{QPHI}=0$ ). They are defined either by the user (IFQQB $=0$ ) or are computed by the program (IFQQB $=1,2$ ).

The flavours and four-momenta of the quark-antiquark pair are stored in

COMMON/FSGNRT/KFLIN(2),QUIN $(4,2)$

where $(I=1$ for the quark and $I=2$ for the antiquark)

$\operatorname{KFLIN}(\mathrm{I})=$ flavour (see table 1$)$,

QUIN $(1, I)=$ momentum along the $x$-axis,

QUIN $(2, I)=$ momentum along the $y$-axis,

$\operatorname{QUIN}(3, \mathrm{I})=$ momentum along the $z$-axis,

QUIN $(4, I)=$ energy.

\subsubsection{Subroutine FSDHA}

This routine handles the decay of the FS's produced in each event starting from the initial FS. We remark that the tree mode produces additional FS's whose flavours and four-momenta are stored in

COMMON/ALLFS/NFS,KFL $(2,20), \mathrm{QU}(4,2,20)$

NFS is the number of FS's generated through the decay sequence and KFL(I,J) and QU(K,I,J) have the same meaning as KFLIN(I) and QUIN(K,I) for the Jth FS. 
One after the other, the content of $\operatorname{KFL}(I, J)$ and QU(K,I,J) for the Jth FS is copied in IFL(I) and $\mathrm{Q}(\mathrm{K}, \mathrm{I})$ of

\section{COMMON/FS/IFL(2),Q(4,2),FSMSQ,FS(6)}

and FSMSQ and FS(K) are updated by calling FSUPD.

The a call to FSDCY generates the decay of the pointed FS.

\subsubsection{Subroutine FSUPD}

Starting from the kinematics of the quark-antiquark pair stored in $Q(I, K)$, this routine computes the FS kinematics, FSMSQ and FS(K)

FSMSQ $=$ mass squared,

$\mathrm{FS}(1)=$ momentum along the $x$-axis,

$\mathrm{FS}(2)=$ momentum along the $y$-axis,

$\mathrm{FS}(3)=$ momentum along the $z$-axis,

$\mathrm{FS}(4)=$ energy,

$\mathrm{FS}(5)=$ mass

$F S(6)=$ absolute value of three momentum .

\subsubsection{Subroutine FSDCY}

This routine generates the decay of each FS following the scheme outlined in section 1 and shown in the flow chart of fig. 2.

The program decides between the baryon and the meson modes by calling BBKER(BBKI,BBRT) which supplies

$$
\begin{aligned}
\text { BBKI }= & \text { Prob. } .(F S \rightarrow \text { baryon-antibaryon }) \\
& \text { /Prob. }(\text { FS } \rightarrow \text { hadrons })
\end{aligned}
$$

The probability array $\operatorname{BBRT}(\mathrm{L}), \mathrm{L}=1,2, \ldots, \mathrm{N}$, permits the choice of the decay channel when the baryon mode has been selected. Finally, the routine BARGEN generates the baron-antibaryon pair. The kinematics and identifiers of the decay products are then stored in the array PCB (see the appendix for the format used).

The subroutine TREE(BB) is called in order to decide between chain decay and tree decay when the meson mode has been selected. BB is the normalized tree-mode probability i.e.

BB $=$ Prob.(tree $) /($ Prob.(tree $)+$ Prob.(chain $))$

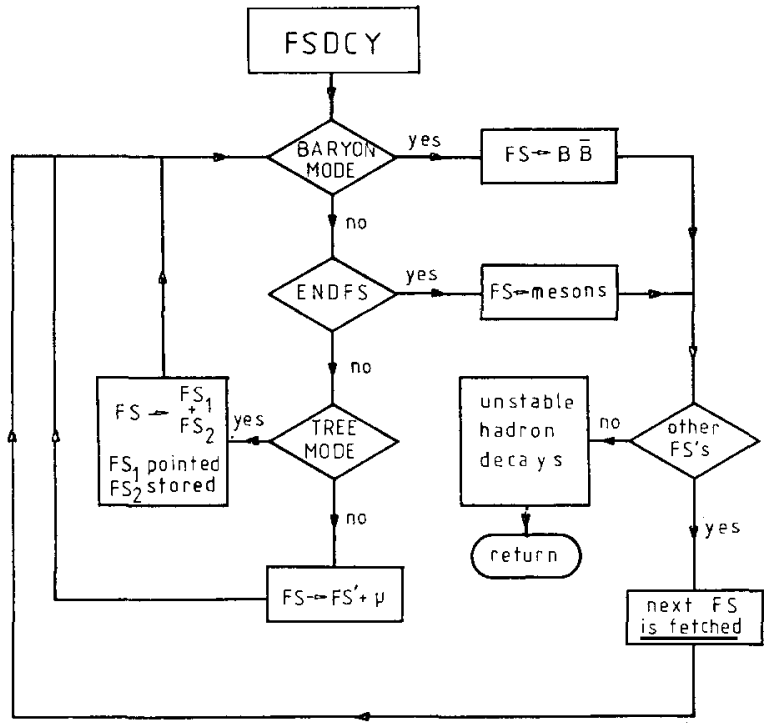

Fig. 2. Flow-chart of subroutine FSDCY.

The action performed by the routine is then:

1) in the chain mode the program generates the chain decay by calling CHAIN(AA) and DCHAIN: the kinematics and identifier of the decay product (vector meson) is stored in the array PRM (see the appendix for the format used).

2) in the tree mode the program generates two FS's by calling TWOFS: one FS is stored in the COMMON/ALLFS/ (NFS is incremented by 1) and the other is stored in COMMON/FS/ becoming the pointed FS.

The program repeates the decay sequence until the FS mass goes below a given threshold which depends on the FS flavours. The program then calls the subroutine ENDFS(IZ), and the FS mass is dumped into low lying mass mesons. The input parameter IZ, with values 1,2 and 3 , identifies three adjacent mass regions whose bounds depend on the FS flavours. This final step is accomplished in the following way. The subroutine ENDFS(IZ) calls ENDFSH(IZ) if at least one heavy flavour is present in the FS; the subroutine ENDFSL(IZ) is called otherwise. These two subroutine generate the FS decay as follows:

ENDFSH(IZ): according to the value of IZ 


$$
\begin{array}{ll}
\text { mass region 1 } & \mathrm{FS} \rightarrow \mathrm{H}+\mathrm{PS} \\
\text { mass region 2 } & \mathrm{FS} \rightarrow \mathrm{H}^{*}+\mathrm{PS} \\
\text { mass region 3 } & \mathrm{FS} \rightarrow \mathrm{H}^{*}+\mathrm{V}
\end{array}
$$

where $\mathrm{H}\left(\mathrm{H}^{*}\right)$ and PS (V) are pseudoscalar (vector) mesons with heavy and light flavours, respectively;

ENDFSL(IZ): according to the value of IZ

mass region $1 \quad \mathrm{FS} \rightarrow 2$ PS or 3 PS

mass region 2 FS $\rightarrow \mathrm{T}+\mathrm{PS}$

mass region $3 \quad \mathrm{FS} \rightarrow 2 \mathrm{~T}$

where PS is any pseudoscalar meson and $\mathrm{T}$ is an excited meson state with mass lying in the mass region 1 , thus decaying accordingly. The kinematics and identifiers of the decay products are stored in the array PCM (see the appendix for the format used).

\subsubsection{Subroutine DCYHDL}

This routine handles the decay of all the unstable hadrons produced by the decay of all FS's in the same event according to the experimental branching ratios [5]. Some of these decays are controlled by the flags in:

\section{COMMON/OPTDCY/IDCY,IDPI0,IDKS, IDLA,IDCHAR,IDBEAU}

with the following meaning:

IDCY $=0$ inhibits the call to subroutine DCYHDL in EPOS,

IDPI0, IDKS, IDLA $=0$ inhibits the decay of $\pi^{0}$, $\mathbf{K}_{\mathrm{s}}^{0}$ and $\Lambda$,

IDCHAR $=0$ inhibits the decay of pseudoscalar charmed mesons,

IDBEAU $=0$ inhibits the decay of pseudoscalar beauty mesons.

These flags have default value $=1$.

The following routines are called by DCYHDL as shown in fig. 3:

PRIDCY generates the decay for all unstable hadrons in the buffer PRM, the decay products are then stored in PCM.

BARYDC similar to PRIDCY except that it acts on PCB.

SECDCY this routine acts on the buffer PCM

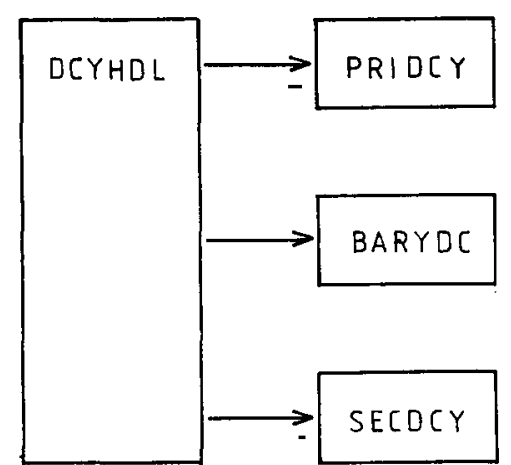

Fig. 3. Flow-chart of subroutine DCYHDL.

and generates all the secondary decays necessary, the result is stored in PCM.

The appendix gives a summary of the "parent identifiers" stored for each particle in PCM.

\subsection{List of subroutines and functions}

Here we give the list of the subroutines and functions used in EPOS with a short description of their actions.

Sub. BARGEN(IC): generates the relevant kinematics and flavours of the baryon-antibaryon pair arising from the FS decay in the decay channel IC.

Sub. BARYDC: generates the decay of the unstable baryons and antibaryons stored in the buffer PCB and fills the buffer PBB and PCM.

Sub. BBBDCY: prints the FS decay channel in the baryon mode.

Sub. BBKER(BBKT,BBK): computes the probability BBKT for the FS decay into a baryon-antibaryon pair and the probability array $\mathrm{BBK}(\mathrm{L})$, $\mathrm{L}=1,2, \ldots, \mathrm{N}$ for each decay channel.

Sub. BEEDCY(I): generates the decay of the beauty flavoured pseudoscalars contained in $\operatorname{PCM}(K, I)$.

Sub. CHADCY(I): generates the hadronic decay of the charm flavoured pseudoscalars contained in PCM(K,I).

Sub. CHAIN(WC): computes the total probability for the chain decay WC and the probability array WGHT(I,L) for the chain decay relative to 
the quark $(I=1)$ and the antiquark $(I=2)$, in the channel L. The array WGHT is stored in COMMON/CHAIN/.

Sub. CHNDCY: prints the FS decay channel in the chain mode.

Sub. CORRAD(ISG): generates the radiative corrections to the initial state and stores the emitted photon (if any) in $\operatorname{PCM}(\mathrm{K}, 1)$.

Sub. DCHAIN(AA): generates the FS chain decay and computes all the relevant kinematics and flavours of the decay products. AA is the total probability of this made.

Sub. DCYHDL: handles the unstable particle decays after the decay of all the FS's generated in each event as shown in fig. 3.

Sub. DEEDCY(I): generates the decay of the charmed pseudoscalar mesons contained in $\operatorname{PCM}(K, I)$.

Sub. DUMPDT: prints all the data used by EPOS.

Sub. ENDDCL: called by DUMPDT.

Sub. ENDDCH: called by DUMPDT.

Sub. ENDDCY: prints the FS decay channels in the ENDFS subroutine.

Sub. ENDFS(IZ): calls either ENDFSH(IZ) or ENDFSL(IZ) according to the presence of a heavy or light flavour, respectively.

Sub. ENDFS1(JP): called by ENDFSL(IZ) to handle the final decay in the mass region 1 . JP is the chosen decay channel.

Sub. ENDFSH(IZ): generates the final step of FS decay in the mass region identified by the input parameter IZ, when the FS is heavy flavoured.

Sub. ENDFSL(IZ): generates the final step of FS decay in the mass region identified by the input parameter IZ, when the FS is light flavoured.

Sub. EPOS: controls the generation of the hadronic final state in the annihilation of electron-positron into hadrons (fig. 1).

Sub. FSDCY: generates the full FS decay sequence as shown in the flow-chart of fig. 2.

Sub. FSDHA: handles the decay of all FS's produced in a single event.

SUb. FSGEN(IP): generates the initial FS kinematics and flavours. IP is returned $=1$ if a photon has been radiated from the initial state, $=0$ otherwise.
Sub. FSUPD: updates the kinematics and flavours of the pointed FS stored in COMMON/FS/.

Sub. FZONE(LQQB,IZONE): finds the appropriate mass region, identified by the index IZONE and flavour LQQB for the final step of FS decay.

Sub. GEN(X,PT,XM,IQ): computes the energy fraction $X$ and the transverse momentum $P T$ (transverse is with respect to the parent FS axis) of the vector meson of mass XM, emitted in the chain mode when either the quark (IQ = 1) or the antiquark $(I Q=2)$ is involved in the decay.

Sub. HEASPC(IDP,IDD,PDAU,PU,PDBAR): spectrum of the leptonic and hadronic decay products of the D and B mesons.

Sub. HEACHA: generates the decay of the pseudoscalar beauty mesons according to the chain decay scheme.

Sub. HYPDCY: prints the baryon decay channels considered in EPOS.

Sub. LAMBDC $(P, K D)$ : generates the decay of the charmed meson $\Lambda_{\mathrm{c}}^{+}$with four-momentum $\mathrm{P}(\mathrm{K})$, $\mathbf{K}=1,2,3,4, \mathbf{K D}$ is the pointed to the parent baryon.

Sub. MESDCY: prints the meson decay channels considered in EPOS.

Sub. OMPHI(WW): computes the weight for the $\omega$ or $\phi$ decay, when they are transversely polarized.

Sub. POLAR(XY,Z,THETA,PHI): computes the polar coordinates (P,THETA, PHI) starting from the Cartesian coordinates $(\mathrm{X}, \mathrm{Y}, \mathrm{Z})$.

Sub. PRIDCY: generates the decay of the vector mesons contained in the buffer PRM and fills the buffer PCM with their decay products.

Sub. PTNAME(ID,STR): gives the name (ASCI string STR) of the particle having identifier ID.

Sub. RADIAT(PG,CTHG,PHIG,ISG): generates the emission of a radiative photon in the initial state from the positron (ISG $=1$ ), from the electron (ISG $=-1$ ); PG is the energy, CTHG and PHIG are the cosine of the polar angle and the azimuthal angle of the emitted photon. If ISG $=0$ no photon is emitted.

Sub. ROTAT(THETA,PHI,V): rotates the three-vector $\mathrm{V}(\mathrm{K})$ bringing the $z$-axis in the (THETA,PHI) direction; the result is returned in $\mathrm{V}(\mathrm{K})$. 
Sub. SECDCY: generates the decays of the unstable mesons contained in the buffer PCM.

Sub. START: computes energy dependent parameters and is called every time the centre-ofmass energy is changed.

Sub. TGEN(X1,X2,CHI,XMFS2): computes the kinematical quantities $\mathrm{X} 1, \mathrm{X} 2, \mathrm{CHI}$ for the tree mode when the parent FS has squared mass XMFS2.

Sub. TREE(WT): computes the total probability for the FS tree mode.

Sub. TWOFS: generates the FS tree decay and determines the relevant kinematics and flavours of the produced FS's. One FS becomes the pointed FS and is stored in COMMON/FS/, the other is stored in COMMON/ALLFS/.

Sub. VORTHO(A,VL,VT,V): generates the vector $\mathrm{V}$ having parallel (VL) and transverse (VT) component with respect to the vector $A$. The transverse component is isotropically generated.

Funct. FRWG(ID): computes the mass of the particle with identifier ID using a Breit-Wigner shape.

Double precis. funct. DRN(XX): double precision random number generator.

Funct. $\mathrm{F}(\mathrm{X} 1, \mathrm{X} 2, \mathrm{CHI})$ : used in the tree mode.

Double precis. funct. $\mathrm{FCG}(\mathrm{S}, \mathrm{XM} 2)$ : polar angle distribution of the hard radiative photon.

Funct. $\mathrm{FCN}(\mathrm{X}, \mathrm{I})$ : called by subroutine HEASPC.

Funct. FCNCG(X,I): used in the radiative hard photon spectrum generation.

Funct. FCNK(X,I): used in the radiative hard photon spectrum generation.

Funct. $\mathrm{FCl}(\mathrm{X})$ : used in the chain mode.

Funct. FC2(X): used in the chain mode.

Funct. FC3(X): used in the chain mode.

Funct. $\operatorname{FEXP}(X)$ : underflow-free definition of $\operatorname{EXP}(-X)$ when $X$ is large and positive.

Double precis. funct. $F H(K)$ : radiative hard photon spectrum.

Funct. FROG1(X,I): used in the tree mode.

Funct. FROG2(X,I): used in the tree mode.

Funct. GG(AM,K,N): is used by BBKER and computes the form factor for the FS baryon mode. AM is the FS mass, $\mathrm{K}$ describes the FS flavours and $\mathrm{N}$ the decay channel.

Funct. HINT(IA): used in the chain mode.
Funct. ICHARG(IC): returns the charge of the particle with identifier ID.

Funct. PCMSQ(A,B,C): computes the square of the centre-of-mass three-momentum for a body of mass A decaying into two bodies of masses $B$ and C.

Double precis. funct. PIHINT(S): hadronic and leptonic vacuum polarization.

Funct. PHSP(AM,X,Y): computes the two body phase space factor with AM decaying mass and $X$ and $\mathrm{Y}$ decay product masses.

Funct. SIG(S,XM2): total cross section for $\mathrm{q}-\overline{\mathrm{q}}$ pair production. $S$ is the square of the centre-ofmass energy and XM2 is the square of the quark mass.

Funct. $\operatorname{SIGDF}(\mathrm{Z})$ : angular distribution $(\mathrm{Z}=$ $\cos \theta$ ) of the initial $\mathrm{q}-\overline{\mathrm{q}}$ pair.

Funct. WQUARK(X): called from subroutine HEASPC.

Funct. WTNEW(U,V,FSM): used in tree mode.

Funct. XMASS(ID): returns the mass of the particle with identifier ID.

The program makes also use of the following subroutines and functions of the CERN Computer Library: RZERO(C205), GAUSS(D103), MIVAR(D509), VZERO(F121), LORENF(U101), LORENB(U101), RNDM(V104), UCOPY(V301), GENBOD(W515).

\section{Input and output}

The user can define the input parameters in the COMMON/EPIN/ and the COMMON/ OPTDCY / as extensively explained in subsections 2.1.1. and 2.1.5.

Then the statement CALL EPOS fills the:

COMMON/EPOUT/ECMR, NTOT,

$\operatorname{PCM}(7,400)$

ECMR is the electron-positron centre-of-mass energy after radiative emission. NTOT is the total number of particles in the final state. $\operatorname{PCM}(\mathrm{K}, \mathrm{I})$ contains the kinematics and identifiers of the Ith particle, as illustrated in subsection 2.1.5 and the appendix.

More information about the primary particles is 
stored in

COMMON/PRIHAD/NPR,PRM(7.75),LBB,

$$
\operatorname{PCB}(7,40)
$$

where NPR is the number of primary mesons in $\mathrm{PRM}$ and LBB is the number of primary baryons and antibaryons in PCB.

The program makes use of a large amount of data stored in BLOCK DATA FIRST, SECOND

Table 2

Particle identifier

\begin{tabular}{|c|c|c|c|c|c|}
\hline Ident. & Part. & Ident. & Part. & Ident. & Part. \\
\hline 1 & $\rho^{+}$ & 101 & $\mathbf{P}$ & 201 & $\mathrm{e}^{-}$ \\
\hline 2 & $\boldsymbol{\rho}^{0}$ & 102 & $\mathbf{n}$ & 202 & $v_{\mathrm{e}}$ \\
\hline 3 & $\rho^{-}$ & 103 & $\Lambda$ & 203 & $\mu^{-}$ \\
\hline 4 & $\mathrm{~K}_{\mathrm{o}}^{*}$ & 104 & $\Sigma^{+}$ & 204 & $v \mu$ \\
\hline 5 & $\mathrm{~K}^{*}$ & 105 & $\Sigma^{0}$ & 205 & $\pi^{-}$ \\
\hline 6 & $\mathrm{AK}^{*}{ }_{0}$ & 106 & $\Sigma^{-}$ & 206 & $N \tau$ \\
\hline 7 & $\mathrm{~K}^{*}$ & 107 & $\Xi^{0}$ & & \\
\hline 8 & $\omega$ & 108 & $\Xi^{-}$ & & \\
\hline 9 & $\phi$ & 109 & $\Delta^{++}$ & & \\
\hline 10 & $\pi^{+}$ & 110 & $\Delta^{+}$ & & \\
\hline 11 & $\pi^{-}$ & 111 & $\Delta^{0}$ & & \\
\hline 12 & $\pi^{0}$ & 112 & $\Delta^{-}$ & & \\
\hline 13 & $v^{\prime}$ & 113 & $\Sigma^{*}{ }_{+}$ & & \\
\hline 14 & $\gamma$ & 114 & $\bar{\Sigma}^{*}{ }_{0}^{+}$ & & \\
\hline 15 & $v$ & 115 & $\Sigma^{*}$ & & \\
\hline 16 & $\mathrm{~K}^{+}$ & 116 & $\Xi{ }_{0}$ & & \\
\hline 17 & $\mathrm{~K}^{-}$ & 117 & $\Xi *$ & & \\
\hline 18 & $\mathbf{K}^{0}$ & 118 & $\Omega^{-}$ & & \\
\hline 19 & $\mathrm{AK}^{0}$ & 119 & $\Lambda_{c}^{+}$ & & \\
\hline 20 & $\mathrm{D}^{*}{ }_{0}$ & 120 & $\Sigma_{c}^{++}$ & & \\
\hline 21 & $\mathrm{D}^{*}{ }_{+}$ & 121 & $\Sigma_{c}^{+}$ & & \\
\hline 22 & $\mathrm{AD}^{+}{ }_{0}^{+}$ & 122 & $\Sigma_{c}^{0}$ & & \\
\hline 23 & $\mathrm{D}^{*}$ & 123 & $\Xi_{c}^{+}$ & & \\
\hline 24 & $\mathrm{D}^{\mathrm{o}^{-}}$ & 124 & $\Xi_{c}^{0}$ & & \\
\hline 25 & $\mathrm{D}^{+}$ & 125 & $\Xi_{\mathrm{c}} \mathrm{a}^{+}$ & & \\
\hline 26 & $\mathrm{AD}^{0}$ & 126 & $\Xi_{c} a^{0}$ & & \\
\hline 27 & $\mathrm{D}^{-}$ & 127 & $\Omega_{c}^{0}$ & & \\
\hline 28 & $\mathrm{~B}^{*}{ }_{0}$ & 128 & $\Sigma_{c}^{*}{ }_{c}^{++}$ & & \\
\hline 29 & $\mathrm{~B}^{*}{ }_{+}$ & 129 & $\Sigma_{c}^{*^{+}}$ & & \\
\hline 30 & $\mathrm{AB}^{+}{ }_{0}^{+}$ & 130 & $\Sigma^{* 0}$ & & \\
\hline 31 & $\mathbf{B}^{*}$ & 131 & $\underline{E *+}$ & & \\
\hline 32 & $\mathrm{~B}^{0^{-}}$ & 132 & $\Xi_{c}^{*} 0$ & & \\
\hline 33 & $\mathrm{~B}^{+}$ & 133 & $\bar{\Omega}_{c}^{* 0}$ & & \\
\hline 34 & $\mathrm{AB}^{0}$ & \multirow{2}{*}{\multicolumn{4}{|c|}{$\begin{array}{l}\text { negative identifier for antibaryons } \\
\text { and antileptons }\end{array}$}} \\
\hline 35 & $\mathrm{~B}^{-}$ & & & & \\
\hline
\end{tabular}

and THIRD. They are derived either from experimental information [5] or from isotopic spin and unitary symmetry requirements. The complete list of the data can be obtained with the statement CALL DUMPDT.

\section{Appendix}

The kinematics and identifiers relative to particle $I$ are stored in the arrays $\operatorname{PRM}(\mathrm{K}, \mathrm{I}), \mathrm{PCB}(\mathrm{K}, \mathrm{I})$ and $\operatorname{PCM}(K, I)$ according to the following format:

$\mathrm{K}=1: \quad$ momentum along the $x$-axis,

$\mathrm{K}=2: \quad$ momentum along the $y$-axis,

$\mathrm{K}=3$ : momentum along the $z$-axis,

$\mathrm{K}=4$ : energy,

$\mathrm{K}=5:$ mass,

$K=6$ : identifier (see table 2).

$\operatorname{PRM}(7, \mathrm{I})=$ flavour identifier of the parent quark,

$\operatorname{PCB}(7, \mathrm{I})=$ flavour identifier of the parent quark,

$\operatorname{PCM}(7, \mathrm{I})=$ pointer to the parent particle array with the following code:

0 when the particle comes from ENDFS,

1-75 when the parent particle is $\operatorname{PRM}(\mathrm{K}, \mathrm{INT}(\operatorname{PCM}(7, \mathrm{I})))$,

101-140 when the parent particle is $\operatorname{PCB}(\mathrm{K}, \mathrm{INT}(\operatorname{PCM}(7, \mathrm{I}))-100)$.

\section{References}

[1] For a recent review, see G. Preparata, in: Physics in Collisions, vol. 1, eds. W.P. Trower and G. Bellini (Plenum, New York, 1982).

[2] G. Preparata and G. Valenti, Nucl. Phys. B183 (1981) 53; Phys. Rev. Lett. 47 (1981) 891.

[3] L. Angelini, L. Nitti, M. Pellicoro, G. Preparata and G. Valenti, Phys. Lett. 119B (1982) 456, 123 B (1983) 246; Phys. Rev. D27 (1983) 1668.

[4] L. Angelini, L. Nitti, M. Pellicoro, G. Preparata and G. Valenti, Riv. Nuovo Cimento 6 (1983) 1.

[5] Review of Particle Properties, Phys. Lett. B111 (1982). 
TEST RUN OUTPUT

FRE-STRING CHAIN DECAY

$\begin{array}{llll}\text { PARENT MASS CHARGE } & \text { DECAY PRODUCTS } \\ \text { GUERESCH } & \text { GORDAH }\end{array}$

\begin{tabular}{|c|c|c|c|c|c|c|}
\hline \multirow{2}{*}{$\mathbf{u}$} & .100 & .667 & & & & \\
\hline & & & $\begin{array}{l}\mathrm{g} \\
\mathbf{u}\end{array}$ & 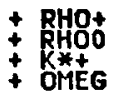 & $\begin{array}{r}.41667 \\
: 20833 \\
: 16667 \\
: 20833\end{array}$ & $\begin{array}{r}1: 5520 \\
1: 5520 \\
1: 7844 \\
1: 5652\end{array}$ \\
\hline \multirow[t]{2}{*}{ D } & .100 & -.333 & & & & \\
\hline & & & $\begin{array}{l}\mathbf{g} \\
\mathbf{5} \\
\mathbf{0}\end{array}$ & $\begin{array}{l}\text { + RHOO } \\
\text { RHO- } \\
+ \text { K*O } \\
+ \text { OMEG }\end{array}$ & $\begin{array}{l}.20833 \\
.41667 \\
.16667 \\
.20833\end{array}$ & $\begin{array}{l}1.5520 \\
1.5520 \\
1.7928 \\
1.5652\end{array}$ \\
\hline \multirow[t]{2}{*}{$\mathbf{s}$} & .469 & -.333 & & & & \\
\hline & & & $\begin{array}{l}\mathbf{D} \\
\mathbf{S}\end{array}$ & $\begin{array}{l}+A K * 0 \\
+K^{*}= \\
+P_{H} I\end{array}$ & $\begin{array}{l}.41667 \\
.41667 \\
.16667\end{array}$ & $\begin{array}{l}1.7926 \\
\frac{1}{2} .7844 \\
1.0392\end{array}$ \\
\hline \multirow[t]{2}{*}{ C } & 1.860 & .667 & & & & \\
\hline & & & $\mathbf{U}$ & $+\underset{D * 0}{D * 0}$ & $\begin{array}{l}.50000 \\
.50000\end{array}$ & $\begin{array}{l}9.0144 \\
4.0202\end{array}$ \\
\hline \multirow[t]{2}{*}{ B } & 4.500 & -.333 & & & & \\
\hline & & & U & $+\underset{A B * 0}{B *}$ & .50000 & $\begin{array}{l}10.5000 \\
10.5000\end{array}$ \\
\hline
\end{tabular}

AU $\quad .100 \quad-.667$

\begin{tabular}{|c|c|}
\hline 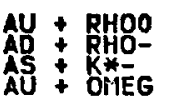 & $\begin{array}{l}.20833 \\
: 41667 \\
: 16667 \\
.20833\end{array}$ \\
\hline
\end{tabular}

$A D \quad .100 \quad .333$

$A U+R H D+\quad: 41667$

1.7926

A5 $\quad .469 \quad .333$

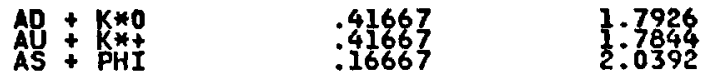

AC $\quad 1.860 \quad-.667$

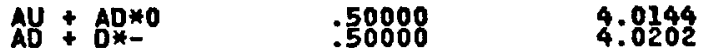

AB $\quad 4.500 \quad .333$

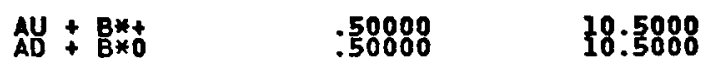


BOUNDS OF THE THREE ENDFS REGIONS

( MASSES ARE IN GEV/C**2)
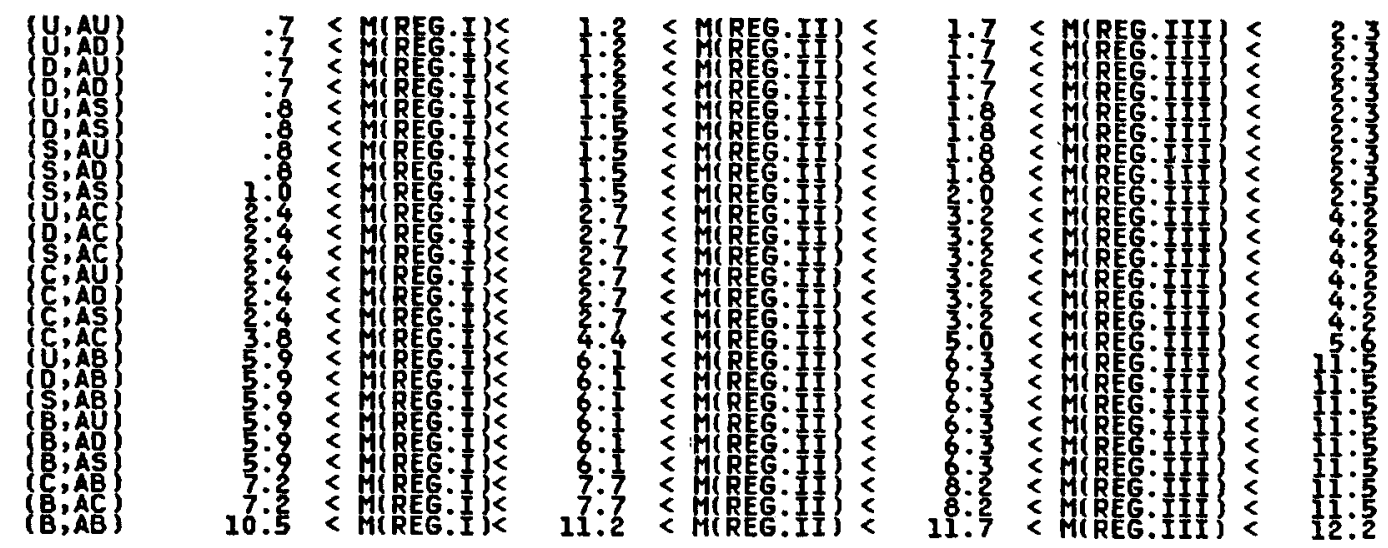

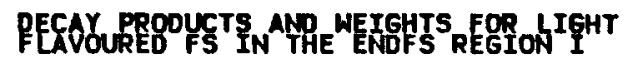

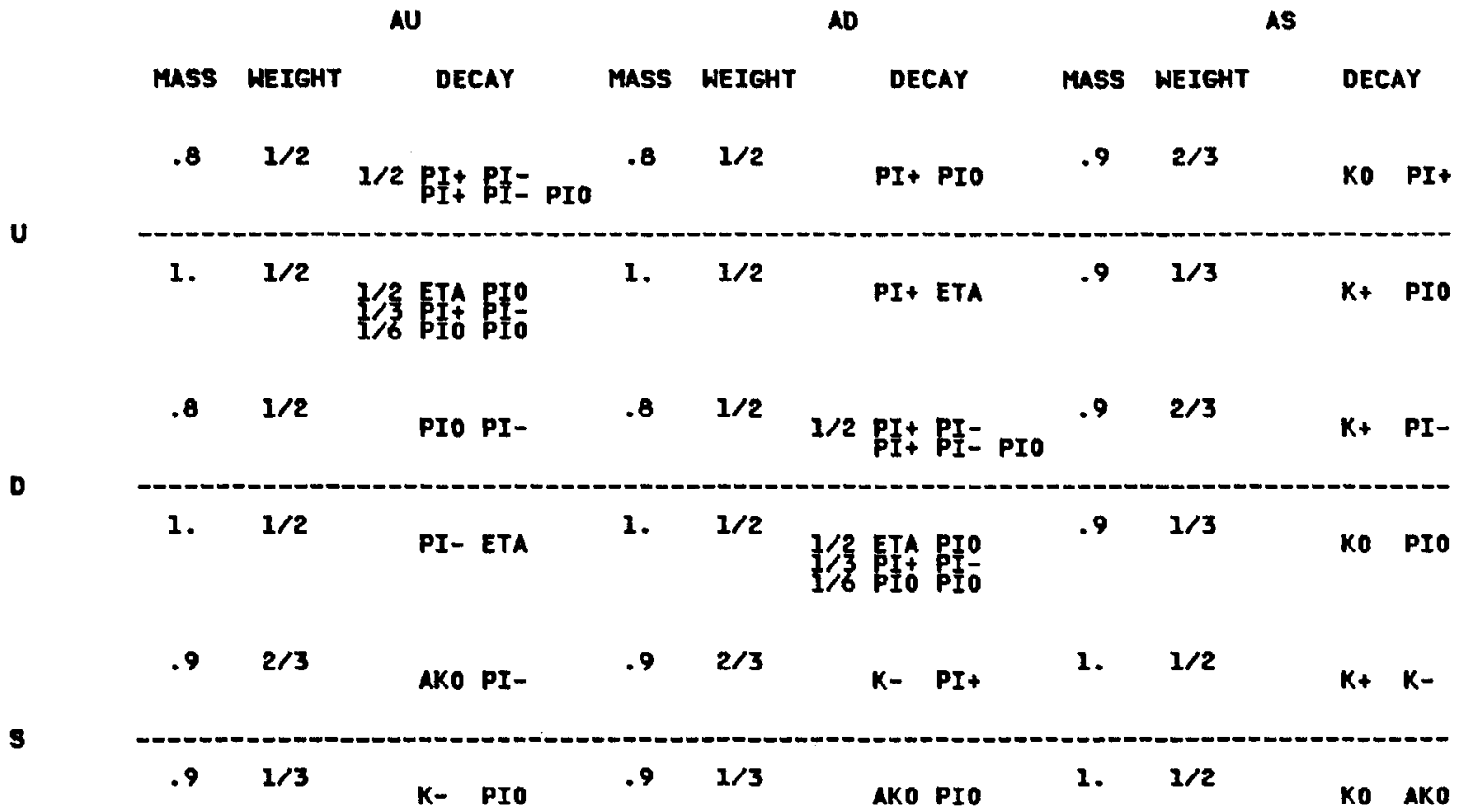

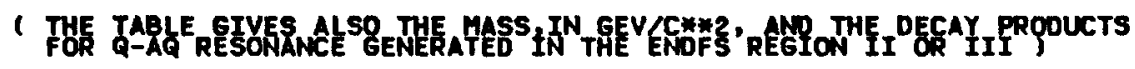




\section{DECAY PRODUCTS ANO MEIGHIS FOR ILIGHT}

AU

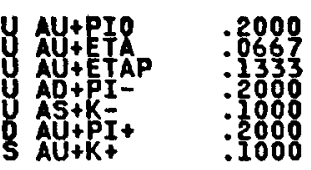

AD
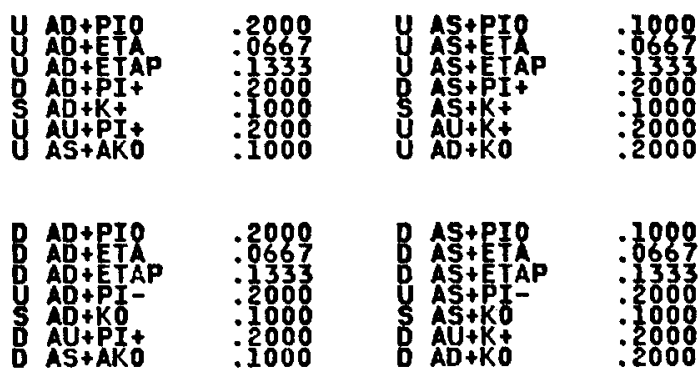

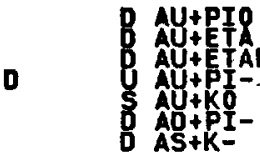
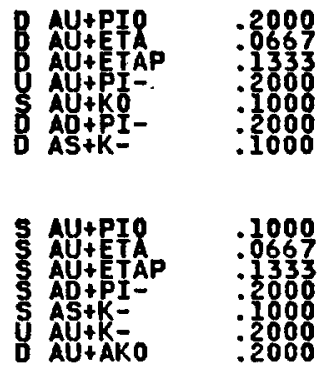

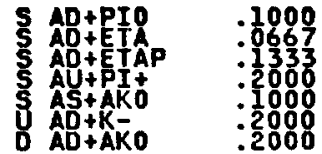




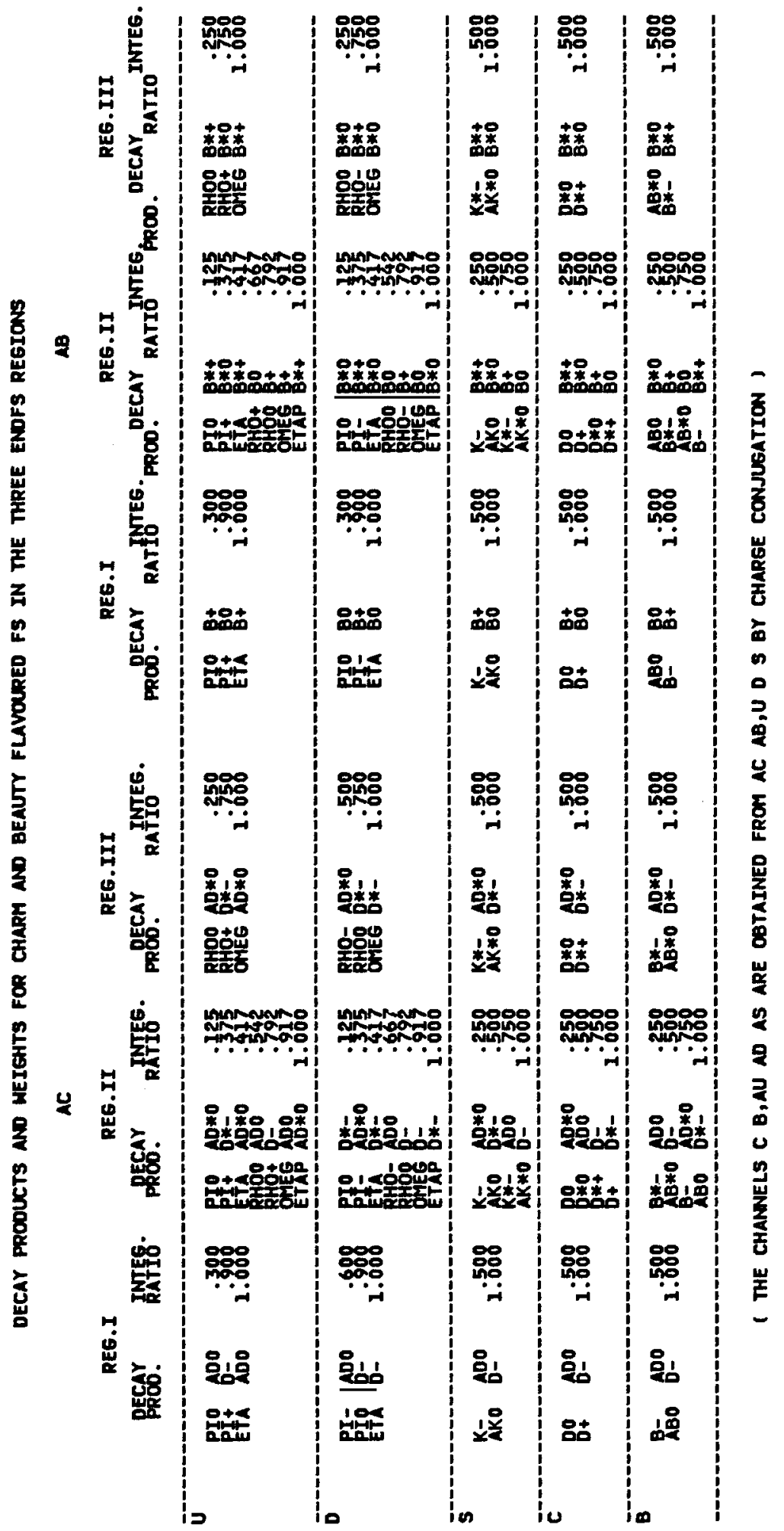


衰

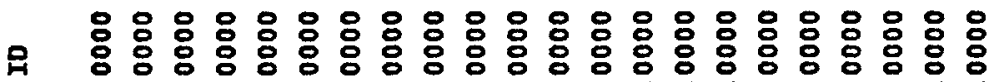

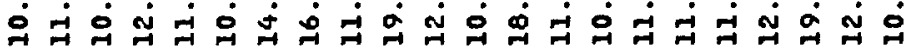

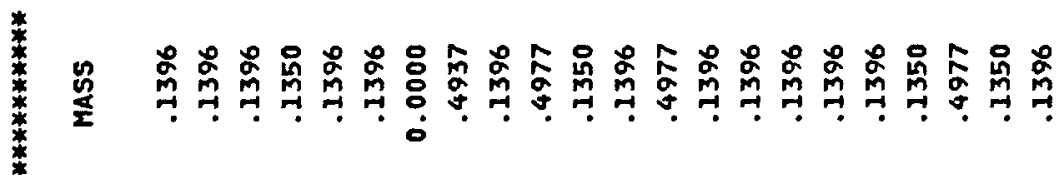

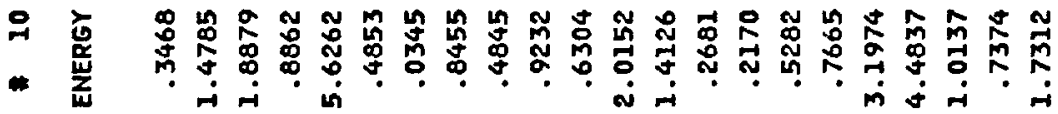

点

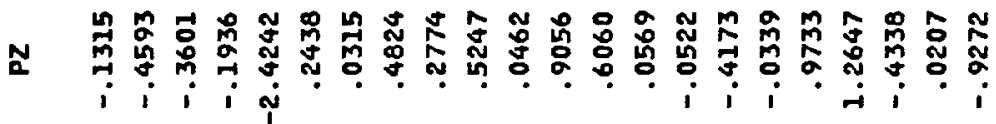

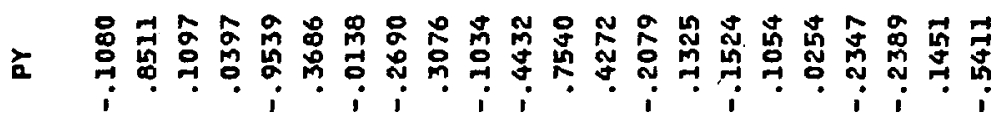

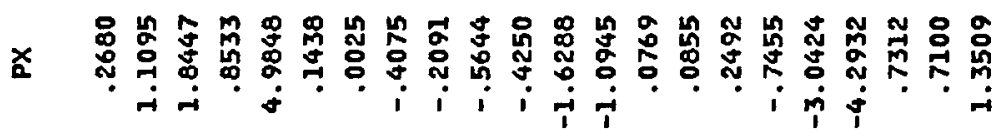




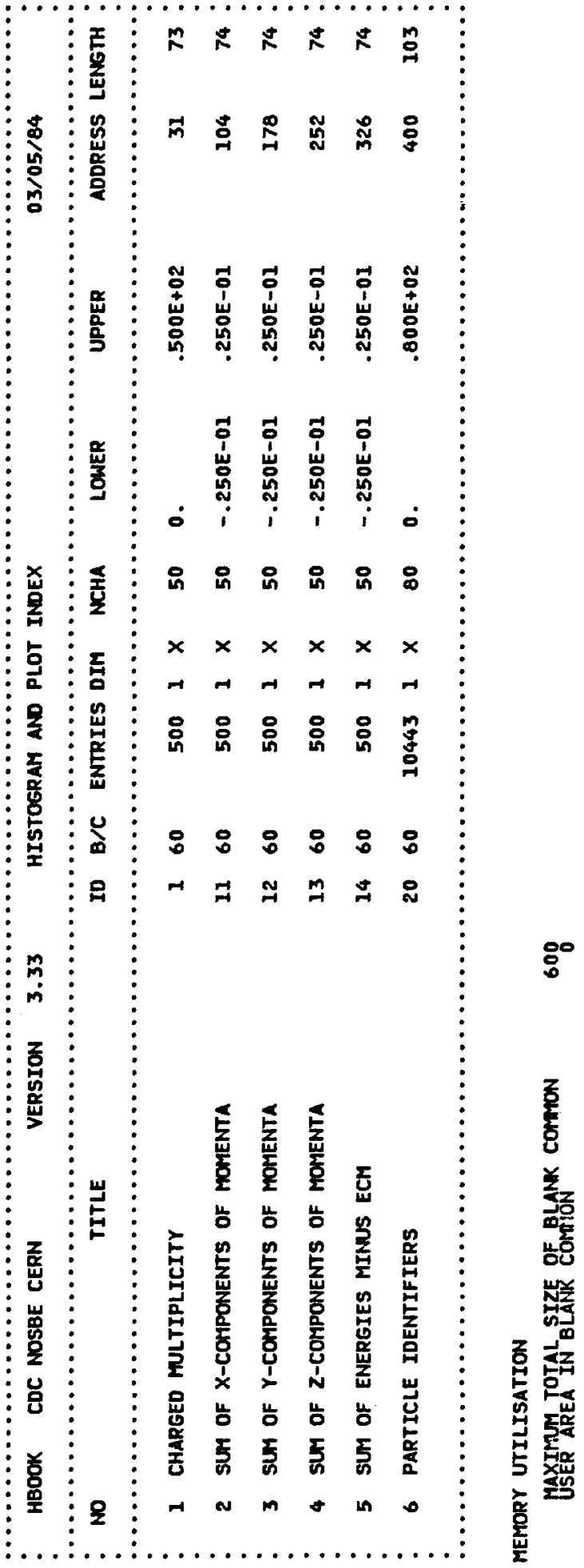




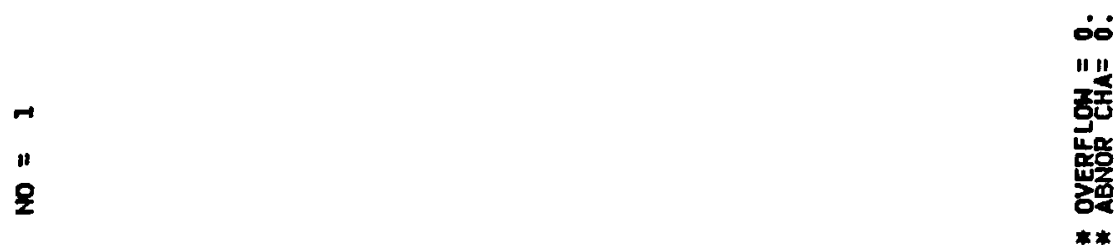

总

$100000000 \times 1000000000000 \times 0$

$\infty \times \infty \times \times \times \times \times \times \times \times$

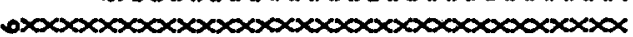

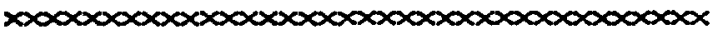

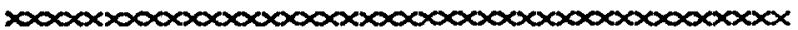
$\times 0 \times 0 \times 0 \times \times \times \times 0 \times 0 \times \times \times \times \times 00000 \times \times \times \times$

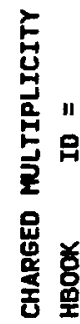
$\infty \times \infty \times \infty \times \infty \times \infty \times \times \infty$ $\Delta \times \infty \times$

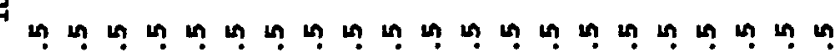

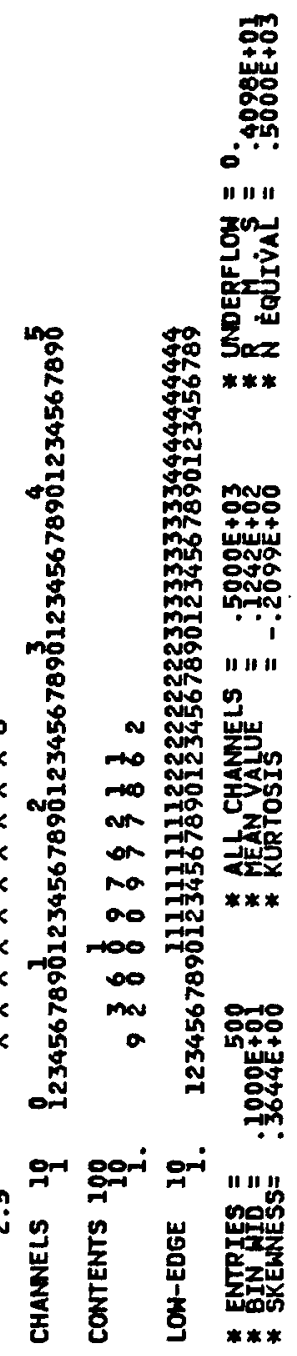

